\title{
NOTE ON THE ZEROS OF FUNCTIONS \\ WITH UNIVALENT DERIVATIVES
}

\author{
MOHAMMAD SALMASSI \\ College of Basic Studies \\ University of Hartford \\ W. Hartford, CT 06117 \\ (Received September 20, 1982 and in revised form July 20, 1983)
}

ABSTRACT. Let $E$ denote the class of functions $f(z)$ analytic in the unit disc $D$, normalized so that $f(0)=0=f^{\prime}(0)-1$, such that each $f^{(k)}(z), k \geq 0$ is univalent in $D$. In this paper we establish conditions for some functions to belong to class E.

KEY WORDS AND PHRASES. Uninalent functions, close-to-convex functions, entire functions. 1980 AMS SUBJECT CLASSIFICATION CODE. 30C45, $30 D 15$.

1. INTRODUCTION.

Let $E$ denote the class of functions analytic in the unit disc $D$, normalized so that $f(0)=0=f^{\prime}(0)-1$, such that $f^{(k)}(z), k \geq 0$ is univalent in $D$. For a survey of E see [1]. In [2] Shah and Trimble proved the following result:

THEOREM A. Let

$$
f(z)=z e^{B z}\left(1-z / z_{1}\right) \text {. }
$$

Suppose

$$
0<\beta \leq 1 / 2,0<z_{1} \leq 2
$$

and

$$
\frac{2+\beta}{1+\beta} \leq z_{1} \leq \frac{2-4 \beta+\beta^{2}}{\beta(2-\beta)}
$$

Then $f(z)$ and all of its derivatives are close-to-convex in D. In particular $f \varepsilon E$. For $B=0.29$,

$$
1.7751 \leq z_{1} \leq 1.8634 \text {. }
$$

2. MAIN THEOREMS.

In this paper we prove the following:

THEOREM 1. Let $f(z)$ be defined by (1.1), suppose that (1.2) holds and $\beta z_{1}<1$. Then;

1 - $f^{\prime}(z)$ is univalent in $|z|<\rho(0<\rho<1)$ if and only if

$$
z_{1} \leq \frac{2+\beta^{2} \rho^{2}-4 \rho \beta}{\beta(2-\beta \rho)} \text {. }
$$

2 - Let $F$ be the class of functions which are derivatives of univalent functions of the from (1.1). For a fixed $\beta$, the radius of univalence of $F, \rho_{F}$, is equal to

$$
\frac{2}{\beta}-\frac{\phi(\beta)+\sqrt{\phi(\beta)^{2}+8}}{2 \beta}
$$


where

$$
\phi(B)=\frac{B(2+B)}{1+B}
$$

THEOREM 2. Let $f(z)$ be defined by (1.1) and suppose that (1.2) holds. If

$$
\frac{2+\beta}{1+\beta} \leq z_{1} \leq \frac{-6+\sqrt{8\left(6-\beta^{2}\right)}}{\beta}
$$

then $f(z), f^{\prime \prime}(z), f^{\prime \prime \prime}(z), \ldots$ are close-to-convex and consequently univalent in $D$. In particular if $B=0.4766,1.6781 \leq z_{1} \leq 1.6791$. In addition, if

$\frac{2+\beta^{2}-4 \beta}{\beta(2-\beta)}<\frac{2+\beta}{1+\beta}$ then $f^{\prime}(z)$ is not univalent in $D$.

THEOREM 3. Let

$$
f(z)=z e^{\beta z}\left(1-z^{2} / z_{1}^{2}\right)
$$

Suppose $0<\beta \leq 0.4$ and

$$
\frac{6+\beta^{2}+6 \beta}{\beta^{2}+2 \beta} \leq z_{1}^{2} \leq \frac{2-6 \beta+3 \beta^{2}}{\beta^{2}}
$$

Then $f(z)$ and all of its derivatives are close-to-convex and consequently univalent in D. In particular for $B=0.2314,3.79664 \leq z_{1} \leq 3.7978$

3. PROOFS.

PROOF OF THEOREM 1. Proof of sufficiency. The function $g(z)=\frac{e^{\beta z}-1}{\beta}$, $\beta$ as in (1.2), is convex in D. If we can show that $\operatorname{Re}\left\{\frac{f^{\prime \prime}(z)}{g^{\prime}(z)}\right\} \leq 0$ for $|z| \leq \rho$ then $f^{\prime}(z)$ will be close-to-convex in $|z| \leq \rho$ and consequently univalent there (see [3]).

If $\phi_{\rho}(x)$ denotes the real part of $\frac{f^{\prime \prime}(z)}{g^{\prime}(z)}$ on $|z|=\rho$, where $x=\operatorname{Rez}$, then

$$
\phi_{\rho}(x)=\left\{2\left(\beta-\frac{1}{z_{1}}\right)+\frac{\beta^{2}}{z_{1}} \rho^{2}\right\}+\beta\left(\beta-\frac{4}{z_{1}}\right) x-\frac{2 \beta^{2}}{z_{1}} x^{2} .
$$

By the maximum principle it suffices to prove that $\phi_{\rho}(x) \leq 0$ for $x$ in $[-1,1]$. For simplicity we write $\phi_{\rho}(x)=a x^{2}+b x+c$. Observe that $b^{2}-4 a c>0$. Thus $\phi_{\rho}(x)$ has two real roots, and we will be done if we can show that

$$
-\rho \geq \frac{-b-b \sqrt{b^{2}-4 a c}}{2 a}
$$

(The larger ront of $\phi_{\rho}(x)$ is $\frac{-b-\sqrt{b^{2}-4 a c}}{2 a}$. See figure 1).

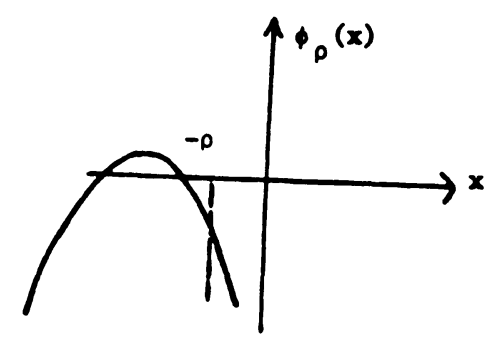

Figure 1 . 
since $a<0,(3.1)$ is equivalent to

$$
\sqrt{b^{2}-4 a c} \leq 2 a p-b .
$$

From the definition of $a$ and $b$ we have

$$
2 a \rho-b=\beta\left[\frac{4}{z_{1}}-\beta\left(1+\frac{4 \rho}{z_{1}}\right)\right]=\beta \frac{4-\beta z_{1}-4 \rho \beta}{z_{1}} \geq \frac{4-1-2}{z_{1}}=\frac{1}{z_{1}}>0
$$

since $\rho \leq 1$ and (1.2) holds.

Squaring both sides of (3.2) and simplifying we get

$$
4 \mathrm{a} \rho(\mathrm{a} p-\mathrm{b}) \geq-4 \mathrm{ac} \text {. }
$$

Divide by $4 \mathrm{a}$ which is negative to get

$$
\rho(b-a \rho) \geq c .
$$

Using the definitions of $a, b$ and $c$, this becomes

$$
z_{1} \beta(\beta \rho-2) \geq 4 \beta \rho-\beta^{2} \rho^{2}-2 \text {. }
$$

From this, noting that $\beta \rho-2<0$, we conclude that (3.2) is equivalent to

$$
z_{1} \leq \frac{2+\beta^{2} \rho^{2}-4 \beta \rho}{\beta(2-\beta \rho)},
$$

which is $(2.1)$.

Proof of necessity. We show that if

$$
z_{1}>\frac{2+\beta^{2} \rho^{2}-4 \beta \rho}{\beta(2-\rho \beta)}
$$

then $f^{\prime \prime}(z)$ has a root in $|z|<\rho$, which means that $f^{\prime}(z)$ is not univalent there. The equation, $f^{\prime \prime}(z)=0$, that is

$$
\frac{-\beta^{2}}{z_{1}} z^{2}+\left(\frac{-4 \beta}{z_{1}}+\beta^{2}\right) z+2 \beta-\frac{2}{z_{1}}=0
$$

has two negative roots given by

$$
\frac{\beta-4 / z_{1} \pm \sqrt{\beta^{2}+8 / z_{1}^{2}}}{\frac{2 \beta}{z_{1}}}
$$

The smaller root lies in the disc $|z|<\rho$ if

$$
\left|\frac{\beta-4 / z_{1}+\sqrt{\beta^{2}+8 / z_{1}^{2}}}{2 \beta / z_{1}}\right|<\rho .
$$

Since the roots are negative (3.4) is equivalent to

$$
-\left(\beta-\frac{4}{z_{1}}\right)-\sqrt{\beta^{2}+8 / z_{1}^{2}}<\frac{2 \beta \rho}{z_{1}}
$$

or

$$
-\beta+\frac{4}{z_{1}}-\frac{2 \beta \rho}{z_{1}}<\sqrt{\beta^{2}+8 / z_{1}^{2}} \text {. }
$$

But

$$
-\beta+\frac{4}{z_{1}}-\frac{2 \beta \rho}{z_{1}}=\frac{4-\beta z_{1}-2 \beta \rho}{z_{1}} \geq \frac{2}{z_{1}}>0
$$

by (1.2) and $\rho \leq 1$. Squaring both sides of (3.5) and simplifying we get (3.3).

This proves the first part of the theorem. To prove the second part note that by definition, $\rho_{F}$ is the largest number such that $g\left(\rho_{F} z\right)$ is univalent for all $g \in F$ in $D$. Let $g \in F$. Then $g=f^{\prime}$ for some $f$ of the form (1.1). In [2] it is shown that $f$ is univalent in $D$, given (1.2), if and only if $z_{1} \geq \frac{2+\beta}{1+\beta} . \rho_{g}$, the radius of univalence of 
$g$, is non zero because $f^{\prime \prime}(0) \neq 0$. Therefore, by the first part of the theorem, the condition

$$
\frac{2+\beta}{1+\beta} \leq z_{1} \leq \frac{2+\beta^{2} \rho_{g}-4 \rho_{g} \beta}{\beta\left(2-\beta \rho_{g}\right)}
$$

is the necessary and sufficient condition for $f(z)$ and $g\left(\rho_{g} z\right)$ to be univalent in $D$. Let $x=2-\rho_{g} \beta$. It follows form (3.6) that

$$
x^{2}-\phi(\beta) x-2 \geq 0
$$

which is true if and only if

$$
x \geq \frac{\phi(\beta)+\sqrt{\phi(\beta)^{2}+8}}{2}
$$

or if

$$
\rho_{g} \leq \frac{2}{\beta}-\frac{\phi(\beta)+\sqrt{\phi(\beta)^{2}+8}}{2 \beta}
$$

The case of equality in (3.7) corresponds to the case where both inequalities in (3.6) are equalities. That is the radius of univalence of the $g$ for which $z_{1}=\frac{2+\beta}{1+\beta}$ is precisely the expression on the right of (3.7). This proves the second part of the theorem.

Note that $\rho_{F}=1$ corresponds to $B \doteq .29$. Also if $B=0.4746, \rho_{F} \doteq 0.2793$.

PROOF OF THEOREM 2. We will show that $\operatorname{Re}\left\{\frac{f^{\prime}(z)}{e^{\beta z}}\right\} \geq 0$ and $\operatorname{Re}\left\{\frac{f^{(n)}(z)}{e^{\beta z}}\right\} \leq 0$ for $n \geq 3$ and $z$ in D. This will show that $f(z)$ and $f^{(n)}(z), n \geq 2$ are close-to-convex in $D$. In [2] it was shown that, if $(1.2)$ holds and $\frac{2+B}{1+B} \leq z_{1} \leq \overline{2}$, then $\operatorname{Re}\left\{\frac{f^{\prime}(z)}{e^{\beta z}} \geq 0\right.$ in $D$. Thus we need only show that $\operatorname{Re}\left\{\frac{\mathrm{f}^{(\mathrm{n})}(\mathrm{z})}{\mathrm{e}^{\beta z}}\right\} \leq 0$ for $\mathrm{n} \geq 3$ in $D$.

If we denote the real part of $\frac{f^{(n)}(z)}{e^{\beta z}}$ on the unit circle for $n \geq 3$ by $\phi_{n}(x)$ where $x=\operatorname{Rez}$, it will be sufficient to show that $\phi_{n}(x) \leq 0$ for $x$ in $[-1,1]$.

Henceforth we assume that $n \geq 3$ and note that

$$
\phi_{n}(x)=n \beta^{n-2}\left(\beta-\frac{n-1}{z_{1}}\right)+\frac{\beta^{n}}{z_{1}}+\beta^{n-1}\left(\beta-\frac{2 n}{z_{1}}\right) x-\frac{2 \beta^{n}}{z_{1}} x^{2} .
$$

The quadratic $\phi_{n}(x)$ will be nonpositive for all $x \varepsilon[-1,1]$ if its discriminant is nonpositive. (We may note that the case when $\phi_{n}(x)$ has two real roots is not of interest). Thus we have

$$
B^{2} z_{1}^{2}+8 \beta^{2} \leq 4 n\left[n-\left(2+B z_{1}\right)\right], \quad n \geq 3 .
$$

This inequality will be satisfied if it is satisfied for $n=3$, that is, if

$$
\beta^{2} z_{1}^{2}+12 \beta z_{1}+8 \beta^{2}-12 \leq 0 .
$$

This holds when $z_{1} \leq \frac{-6+\sqrt{8\left(6-\beta^{2}\right)}}{\beta}$, which is true by $(2.2)$.

Letting $\beta=0.4746$, calculations show that (2.2) implies $1.6781 \leq z_{1} \leq 1.6791$.

Finally if $\frac{2+\beta^{2}-4 \beta}{\beta(2-\beta)}<\frac{2+\beta}{1+\beta}$, then $z_{1}>\frac{2+\beta^{2}-4 \beta}{\beta(2-\beta)}$ by $(2.2)$. Thus if $\beta z_{1}<1$, then by the first part of Theorem $1 f^{\prime}(z)$ is not univalent in $D$. But if $B z_{1}=1$, then $f^{\prime \prime}(0)=0$ and $\mathrm{f}^{\prime}(\mathrm{z})$ is not univalent in $D$.

PROOF OF THEOREM 3. Note that $\frac{3-\sqrt{3}}{3} \doteq 0.4226$ is the smaller zero of $2-6 \beta+3 \beta^{2}$. Thus $B \leq 0.4$ guarantees that the rightmost expression in (2.4) is positive. Let $a=\frac{1}{z_{1}^{2}}$ and 
$\phi_{n}(x)=\operatorname{Re}\left\{\frac{f^{(n)}(z)}{e^{\beta z}}\right\}$ on the unit circle where $x=\operatorname{Rez}$. We will prove the theorem by showing that $\phi_{1}(x) \geq 0, \phi_{2}(x) \geq 0$ and $\phi_{n}(x) \leq 0$ for $n \geq 3$ and $x$ in $[-1,1]$.

First observe that

$$
\phi_{1}(x)=-4 a \beta x^{3}-6 a x^{2}+(3 a \beta+\beta) x+1+3 a
$$

and

$$
\phi_{1}^{\prime}(\mathrm{x})=-12 \mathrm{a} \beta \mathrm{x}^{2}-12 \mathrm{ax}+3 \mathrm{a} \beta+\beta \text {. }
$$

We will have $\phi_{1}(-1) \geq 0$ and $\phi_{1}(1) \geq 0$ if $\frac{1-\beta}{3-\beta} \geq a$ and $\frac{\beta+1}{3+\beta} \geq a$, respectively. But both inequalities are true; this follows from (2.4) and the fact that, for $B \leq 0.4$, $\frac{\beta+1}{\beta+3}>\frac{1-\beta}{3-\beta}>\frac{2 \beta+\beta^{2}}{6+\beta^{2}+6 \beta} \cdot \Phi_{1}^{\prime}(x)$ has one positive and one negative root. Also, since $\dot{\phi}_{1}^{\prime}(-\mathrm{x})=-9 \mathrm{a} \beta+12 \mathrm{a}+\beta=\mathrm{a}(12-9 \beta)+\beta>0$,

the negative root of $\phi_{1}{ }^{\prime}(x)$ lies to the left of -1 . (See Figure 2).

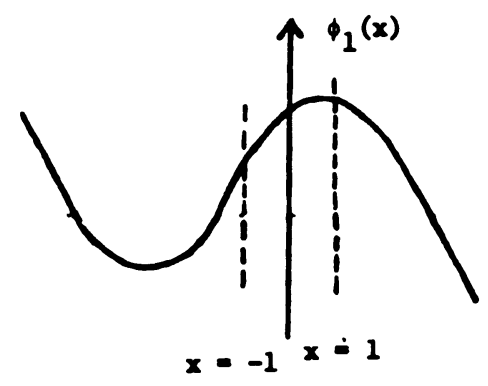

Figure 2.

Thus $\phi_{1}(x) \geq 0$ for $x$ in $[-1,1]$. Next note that

$$
\phi_{2}(x)=-4 a \beta^{2} x^{3}-12 a \beta x^{2}+\left(3 a \beta^{2}-6 a+\beta^{2}\right) x+2 \beta+6 a \beta \text {. }
$$

Because of (2.4) and the fact that $\frac{\beta^{2}}{2-6 \beta+3 \beta^{2}}>\frac{\beta^{2}}{3\left(2-\beta^{2}\right)}$ for $\beta \leq .4$, the coefficient of $x$ in $\phi_{2}(x)$ is negative. If follows from (2.4) that if $x \varepsilon[0,1]$ we have,

$$
\phi_{2}(x) \geq-4 a \beta^{2}-12 a \beta+3 a \beta^{2}-6 a+\beta^{2}+2 \beta+6 a \beta=\beta^{2}+2 \beta-a\left(6+\beta^{2}+6 \beta\right)>0 .
$$

Similarly for $x$ in $[-1,0)$

$$
\phi_{2}(x)>-12 a \beta+2 \beta+6 a \beta=2 \beta(1-3 a) \text {. }
$$

But $1-3 a>0$; this follows from $\frac{1}{3}>\frac{2 \beta+\beta^{2}}{6+\beta^{2}+6 \beta}$ and $(2.4)$. Consequently $\phi_{2}(x) \geq 0$ for $x$ in $[-1,1]$.

From now on we assume that $\mathrm{n} \geq 3$. Note that

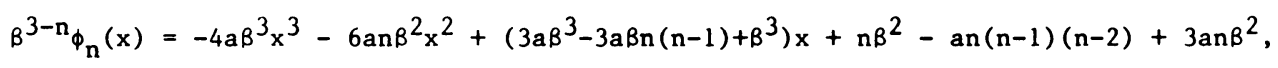


and

$$
\beta^{2-n_{\phi_{n}}}(x)=-12 a \beta^{2} x^{2}-12 a n \beta x+3 a \beta^{2}-3 a n(n-1)+\beta^{2}
$$

Since $3 a \beta^{2}-3 a n(n-1)+\beta^{2}<0, \varphi_{n}^{\prime}(x)$ has two negative roots. Let $t$ denote the larger of the roots. If we can show that $\phi_{n}(-1) \leq 0$ and $-1 \geq t$, then the graph of $\phi_{n}$ will be as in Figure 3, and accordingly $\phi_{n}(x) \leq-1$ for $x$ in $[-1,1]$.

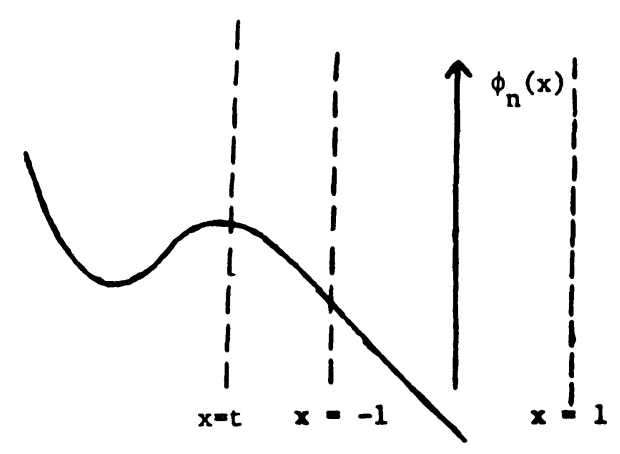

Figure 3.

But

$$
\beta^{3-n_{\phi}}(-1)=\beta^{3}(a-1)+n\left[-3 a \beta^{2}+\beta^{2}+3 a \beta(n-1)-a(n-1)(n-2)\right] \text {. The expression }
$$

inside the bracket above will be negative for $n>3$ if it is non positive for $n=3$, that is, if

$$
a\left(2-6 \beta+3 B^{2}\right) \geq B^{2} \text {. }
$$

But (3.8) is a consequence of $(2.4)$, if we note that $2-6 \beta+3 \beta^{2}>0$ for $\beta \leq 0.4$.

Moreover a $<1$. Thus $\phi_{n}(-1) \leq 0$.

Now the inequality $-1 \geq t$ is equivalent to

$$
-1 \geq \frac{-6 a n B+\sqrt{36 a^{2} n B^{2}}+36 a^{2} B^{4}+12 a B^{4}}{12 a B^{2}}
$$

which is equivalent to

$$
6 a n B-12 a B^{2} \geq \sqrt{36 a^{2} n B^{2}+36 a^{2} B^{4}+12 a B^{4}} .
$$

Note that the left hand side of (3.9) is positive. Squaring both sides of (3.9) and simplifying, we see that (3.9) is equivalent to

$$
3 a n(n-4 B-1) \geq \beta^{2}(1-9 a) .
$$

This inequality will hold for $n \geq 3$ if it holds $f \circ n=3$, that is, if

$$
a \geq \frac{B^{2}}{9\left(B^{2}-4 \beta+2\right)}-\ldots
$$

But from (2.4) and the fact that $B \leq 0.4$, we have that $\frac{\beta^{2}}{2-6 \bar{B}+3 B^{2}}>\frac{B^{2}}{9\left(B^{2}-4 B+2\right)}$ and (3.10) follows from this.

Finally, letting $B=.2314$, calculations show that (2.4) implies that $3.7964 \leq z_{1} \leq 3.9798$. 
4. REMARKS.

(i) It follows from the proof of the first part of Theorem 1 that if (1.2) holds and $B z_{1}<1$ then the inequality $z_{1} \leq \frac{2-4 \beta+\beta^{2}}{\beta(2-\beta)}$ is the necessary and sufficient condition for $f^{\prime}(z)$ to be close-to-convex in $D$. This along with the fact that, given $(1.2), f(z)$ is close-to-convex if and on $1 y$ if $z_{1} \geq \frac{2+\beta}{1+\beta}$ implies that if (1.2) holds and $\beta z_{1}<1,(1.3)$ is the necessary and sufficient condition for $f(z)$ and $f^{\prime}(z)$ to be close-to-convex in $D$. (ii) If in Theorem 3 we have $z_{1}^{2}=\frac{6+\beta^{2}+6 \beta}{\beta^{2}+2 \beta}$ then $f^{\prime \prime}(1)=0$ in which case $f^{\prime}(z)$ is not univalent in a disc larger than $D$.

(iii) In [4] I have showed that if

$$
f(z)=z e^{\beta z}\left(1-z / z_{1}\right)\left(1-z / z_{2}\right)
$$

and if

$$
\begin{aligned}
& 0<\beta<1 / 3, \quad \beta \leq b \leq 1, \\
& \frac{2 b-2 \beta+4 \beta b-\beta^{2}+b \beta^{2}}{\beta^{2}+6 \beta+6} \geq a, \\
& \quad a \geq \frac{b \beta}{1-3 \beta}, \\
& b-2 \beta-3 a \beta+b \beta-3 a+1 \geq 0,
\end{aligned}
$$

where $a=\frac{1}{z_{1} z_{2}}$ and $b=\frac{1}{z_{1}}+\frac{1}{z_{2}}$, then $f(z)$ and all of its derivatives are close-to-convex in D. If $z_{2}>z_{1}$, and $\beta=0.01$ then calculations show that $z_{1}=2.05$ and $z_{2}=94.9298$ satisfy the above inequalities. If $z_{1}=z_{2}$ and $B=0.08$ then $z_{1}=4.3478$ will satisfy the above inequalities.

(iv) Let $f(z)=z e^{\beta z}\left(1-z / z_{1}\right)$, where $z_{1}=x_{1}+i y_{1}, x_{1} \geq 3 / 2$ and $0<\beta \leq 0.29$. We can show that $f(z)$ and all of its derivative are close-to-convex in $D$ if

and

$$
(B+2) x_{1}+2\left|y_{1}\right|(1+B) \leq(1+B)\left|z_{1}\right|^{2}
$$

$$
B\left[x_{1}(4-\beta)+(2-\beta)\left|z_{1}\right|^{2}+2(2-\beta)\left|y_{1}\right|\right] \leq 2 x_{1} .
$$

When $y_{1} \geq 0$ the region in which $z_{1}$ lies is the shaded region in Figure 4 . (The case $y_{1} \leq 0$ is completely symmetric).

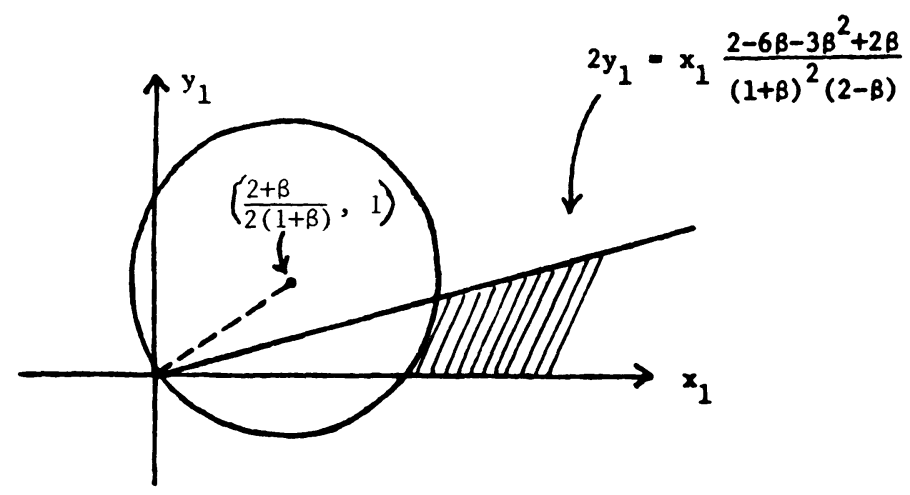

Figure 4 . 
As we see from the picture, the smallest value of $\left|z_{1}\right|$ is obtained when $y_{1}=0$ in which case the above inequalities reduce to (1.3).

ACKNOWLEDGEMENT. The author acknowledges his debt to the referee and Professor S. Y. Trimble for their comments on this paper.

\section{REFERENCES}

1. SHAH, S.M. and TRIMBLE, S.Y. Analytic Functions with Univalent Derivatives, Indian Journal of Mathematics, Vo1. 20, No. 3, Sept. 1978, pp. 265-299.

2. SHAH, S.M. and TRIMBLE, S.Y. On the Zeros of Univalent Functions with Univalent Derivatives, Annali di Mathematica pura ed applicata (IV), Vol. CXXI, PP. 309-317.

3. KAPLAN, W. Close-to-Convex Schlicht Functions, Michigan Math. Jour., 1 (1952), PP. $169-185$.

4. SALMASSI, M. Some Classes of Entire Functions of Exponential Type in One and Several Complex Variables, Doctoral Dissertation 1978, University of Kentucky. 


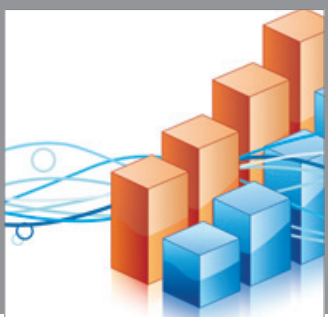

Advances in

Operations Research

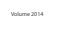

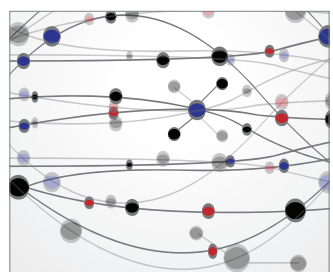

\section{The Scientific} World Journal
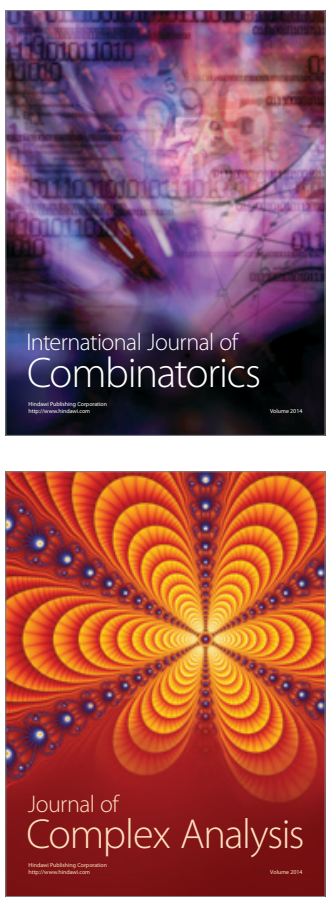

International Journal of

Mathematics and

Mathematical

Sciences
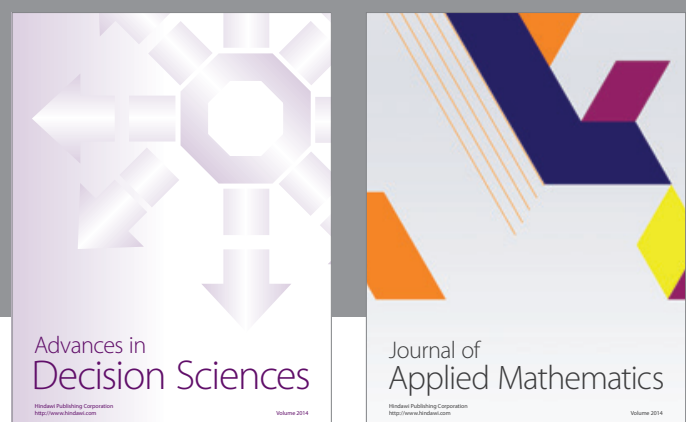

Journal of

Applied Mathematics
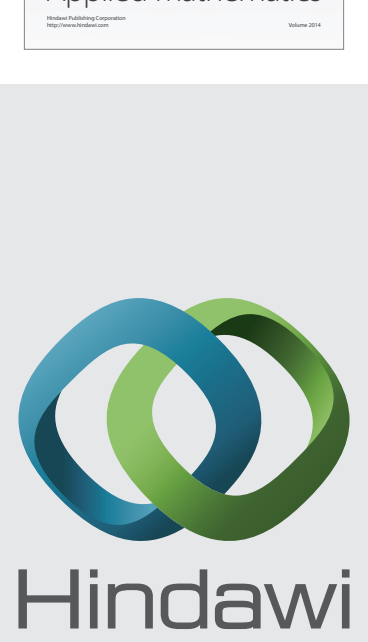

Submit your manuscripts at http://www.hindawi.com
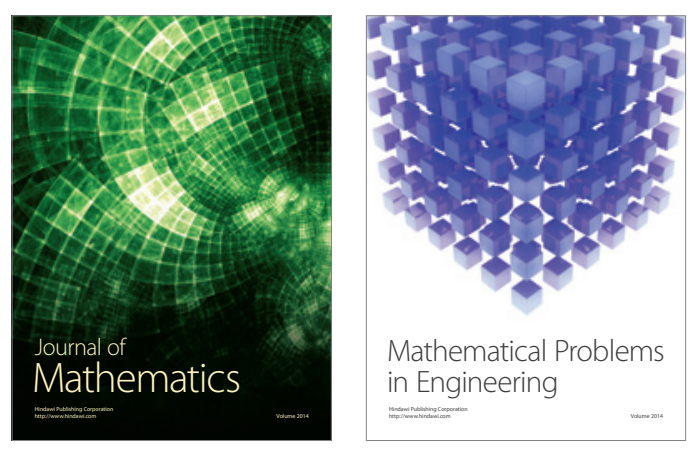

Mathematical Problems in Engineering
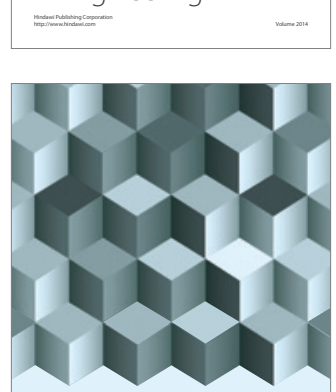

Journal of

Function Spaces
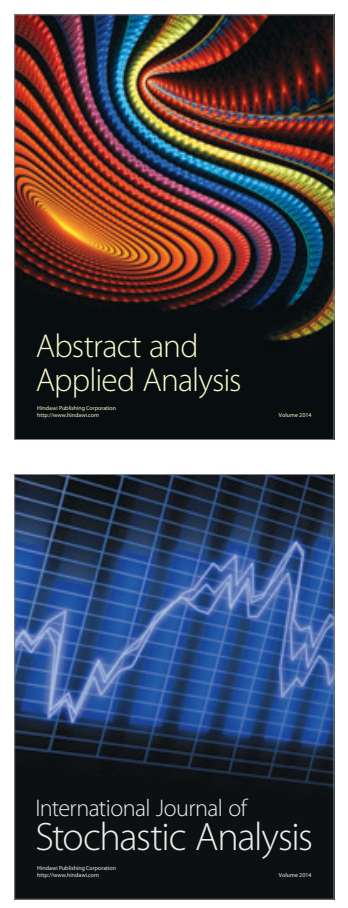

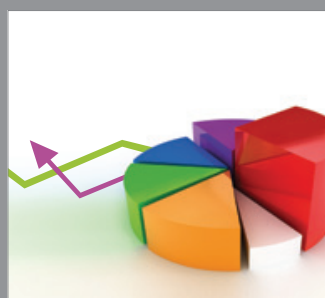

ournal of

Probability and Statistics

Promensencen
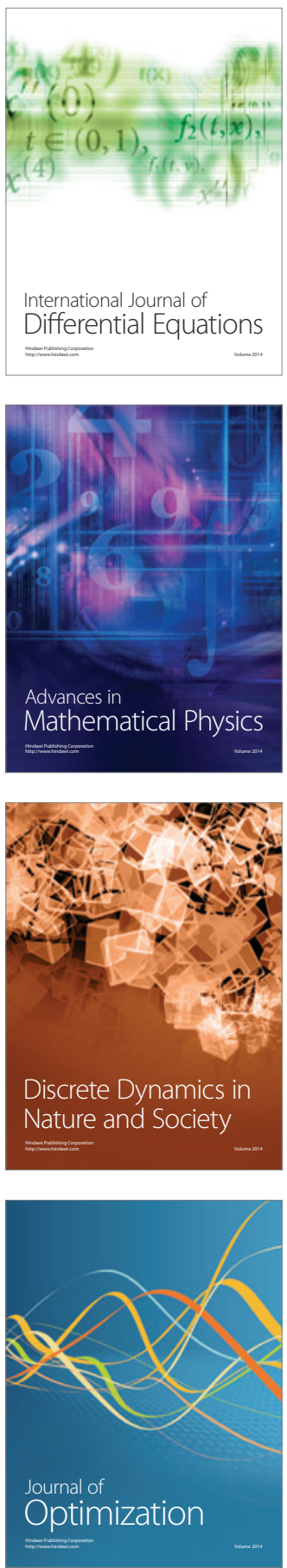